\title{
Ballistic transport in one-dimensional loops with Rashba and Dresselhaus spin-orbit coupling
}

\author{
V. Marigliano Ramaglia, ${ }^{1}$ V. Cataudella, ${ }^{1}$ G. De Filippis,${ }^{1}$ and C. A. Perroni ${ }^{2}$ \\ ${ }^{1}$ Coherentia CNR-INFM and Dipartimento di Scienze Fisiche, Università degli Studi di Napoli “Federico II," 80126 Napoli, Italy \\ ${ }^{2}$ Institute of Solid State Research (IFF), Research Center Jülich, D-52425 Jülich, Germany
}

(Received 4 November 2005; published 25 April 2006)

\begin{abstract}
We discuss the combined effect of Rashba and Dresselhaus spin-orbit interactions in polygonal loops formed by quantum wires, when the electron are injected in a node and collected at the opposite one. The conditions that allow perfect localization (zero transmission coefficient) are found. Furthermore, we investigate the suppression of the transmission coefficient oscillations that appear, in the presence of a magnetic flux, when the electron is injected and collected at the same node. The transmission suppression appears also in the AharonovBohm configuration where the input and the output node are at opposite sides of the loop. Finally, we point out that a recent experimental realization of a ballistic spin interferometer can be used to obtain a reliable estimate of the magnitude ratio of the two spin-orbit interactions.
\end{abstract}

DOI: 10.1103/PhysRevB.73.155328

PACS number(s): 73.23.Ad, 71.70.Ej

\section{INTRODUCTION}

The main goal of the spintronics is the manipulation of spins in semiconductor nanostructures. To this aim, a large number of devices exploiting spin-orbit (SO) interactions ${ }^{1-6}$ has been proposed. The SO interaction relevant in semiconductors are the Rashba effect and the Dresselhaus effect. The former ${ }^{7}$ appears at the interface of semiconductors lacking of structural inversion symmetry, and its magnitude can be controlled by an applied gate voltage. The devices based on this effect use the quantum interference, due to the spin precession, between different paths. Among others, we are reminded of the ballistic spin interferometer, ${ }^{8}$ in which a square loop is followed along a self-intersecting trajectory in a clockwise and anticlockwise way, that, recently, has been used to demonstrate experimentally the occurrence of the spin precession interference phenomenon. ${ }^{9}$ The Rashba SO coupling strength has been measured by using the suppression of the Al'tshuler-Aronov-Spivak oscillations ${ }^{11}$ by Koga et al., ${ }^{9}$ obtaining values in agreement with theoretical estimates and with other weak antilocalization measurements. ${ }^{10}$ Besides, it has been shown that the Rashba effect is also able to induce localization effects in quantum networks. ${ }^{12-14}$

The inversion asymmetry in the bulk semiconductor gives rise to spin-dependent bulk band structure. Consequently at the surface this SO interaction, known as "Dresselhaus term," 15 adds to the Rashba term. Recent measurements based on the spin-galvanic effect, provided the ratio between the magnitude of Rashba and Dresselhaus terms. This ratio can reach values as large as $2.14 \pm 0.25$ in the InAs quantum well. ${ }^{16}$ The Rashba term is in general dominant but the Dresselhaus interaction can have observable effects.

In a quantum wire the two SO couplings yield together a spin precession depending on the angular position of the wire. ${ }^{17}$ In the experiments by Ganichev et al. ${ }^{16}$ a circularly polarized light produces a spin galvanic current whose intensity exhibits an angular dependence that allows the measure of the ratio between the SO couplings. Schliemann et al. ${ }^{18}$ have proposed a spin-field-effect transistor in which the presence of the two SO couplings with equal magnitudes can give polarized currents whose spin does not depend on the momentum. In such a way the spin-independent scattering processes become ineffective in the particular direction in which the spin precession is suppressed.

In this paper we study the interference effects in onedimensional loops due to spin precession when both the two $\mathrm{SO}$ interactions are present. We assume that a single incident wave splits coherently between two paths and then interferes upon recombining at the output. The paper is organized in the following way. In order to be self-contained in Sec. II we recall a number of already known results ${ }^{16,18}$ that will be used to describe the spin precession in a quantum wire under the two SO couplings. ${ }^{23}$ In Sec. III we show how the localization in a polygonal loop can be achieved. We emphasize that for a diamond square loop with the diagonal oriented in [010] crystallographic direction there is a periodic set of values of the SO strengths that gives perfect localization, i.e., the transmission coefficient vanishes. Rotating the diamond square loop the localization is lost. We also show that for particular rhombic and exagonal loops the transmission vanishes only at specific values of the SO strengths. In Sec. IV we consider what happens when a magnetic flux threads the loop, i.e., we analyze a ballistic spin interferometer with both SO couplings. Particular attention will be paid to the suppression of the transmission coefficient oscillations that appear when the input and the output node coincide. We will see how SO magnitudes ratio shifts the values of the Rashba SO strength at which the transmission becomes independent on the magnetic flux. Finally, we prove that the AharonovBohm (AB) oscillations appearing when we inject and collect the current in opposite nodes, can also be modulated varying the two SO couplings. Section $\mathrm{V}$ is dedicated to some concluding remarks.

\section{SPIN PRECESSION DUE TO RASHBA AND DRESSELHAUS COUPLING}

\section{A. The spin-orbit couplings in a two dimensional electron gas}

In order to set the notation, let us be reminded of the eigenstates and the energy eigenvalues of an electron confined in the $x-z$ plane and subjected to both Rashba and 
Dresselhaus spin-orbit interaction. ${ }^{18}$ The Hamiltonian takes the form

$$
H=\frac{\hbar^{2}}{2 m}\left(p_{x}^{2}+p_{z}^{2}\right)+H_{R}+H_{D}
$$

where

$$
H_{R}=\frac{\alpha}{\hbar}\left(\sigma_{z} p_{x}-\sigma_{x} p_{z}\right)
$$

and

$$
H_{D}=\frac{\beta}{\hbar}\left(\sigma_{z} p_{z}-\sigma_{x} p_{x}\right)
$$

are the Rashba and the Dresselhaus interactions, respectively. We have chosen the $x$ axis and $z$ axis in [010] and [100] crystallographic directions, respectively. It is easy to check that

$$
\psi_{\vec{k}, \pm}(x, z)=\exp \left[i\left(k_{x} x+k_{z} z\right)\right]\left|\begin{array}{l}
\cos \nu_{ \pm} \\
\sin \nu_{ \pm}
\end{array}\right|
$$

are eigenfunctions of Eq. (1), with eigenvalues given by

$$
E_{ \pm}=\frac{\hbar^{2}}{2 m} k^{2} \pm \sqrt{\left(\alpha^{2}+\beta^{2}\right) k^{2}+4 \alpha \beta k_{x} k_{z}}
$$

where $k=\sqrt{k_{x}^{2}+k_{z}^{2}}\left(k_{x}=k \cos \theta, k_{z}=k \sin \theta\right)$ is the modulus of the momentum in the $x-z$ plane. In Eq. (4) we have defined

$$
\nu_{ \pm}=\arctan \frac{k_{0} \cos \theta+k_{1} \sin \theta \mp k_{s o}(\theta)}{k_{0} \sin \theta+k_{1} \cos \theta},
$$

where

$$
k_{s o}(\theta)=\sqrt{k_{0}^{2}+k_{1}^{2}+2 k_{0} k_{1} \sin 2 \theta} \text {, with } k_{0}=\frac{m \alpha}{\hbar^{2}} \text { and } k_{1}=\frac{m \beta}{\hbar^{2}} .
$$

We note that there are two values of $k$ corresponding to the same energy $E=\left(\hbar^{2} / 2 m\right) \xi^{2}$ and they are given by

$$
k=k_{ \pm}=\sqrt{\xi^{2}+k_{s o}^{2}} \mp k_{s o}
$$

with the corresponding energy that can be rewritten as

$$
E_{ \pm}=E=\frac{\hbar^{2}}{2 m}\left[k_{ \pm}^{2} \pm 2 k_{ \pm} k_{s o}(\theta)\right] .
$$

The spinors $\chi_{ \pm}$of the two degenerate modes are orthogonal to each other, being

$$
\nu_{-}=\frac{\pi}{2}+\nu_{+}
$$

therefore we have

$$
\chi_{+}=\left|\begin{array}{c}
\cos \nu_{+} \\
\sin \nu_{+}
\end{array}\right| \quad \text { and } \quad \chi_{-}=\left|\begin{array}{c}
-\sin \nu_{+} \\
\cos \nu_{+}
\end{array}\right| .
$$

It is worthy to note that with the only Rashba interaction $\left(k_{1}=0\right)$ we have

$$
\nu_{+}=-\frac{\theta}{2}
$$

We are reminded that the Rashba SO interaction can be viewed as a magnetic field parallel to the plane and orthogonal to the wave vector $\vec{k}$ that orientates the spin along the direction perpendicular to the wave vector. ${ }^{19}$ In particular, when the mode (-) propagates in the $x$ direction the spinor $\chi_{-}=\left|\begin{array}{l}0 \\ 1\end{array}\right|$ is in the spin down state along the $z$ direction. On the other hand, with only Dresselhaus interaction $\left(k_{0}=0\right)$, we have

$$
\nu_{+}=-\frac{\pi}{4}+\frac{\theta}{2}
$$

and the SO magnetic field is opposite to $\vec{k}$. Now, when the mode (-) propagates in the $x$ direction, $\chi_{-}=\left.(1 / \sqrt{2})\right|_{1} ^{1} \mid$ and the spin is oriented along the $x$ axis. When both the SO interactions are present, the effective SO magnetic field, parallel to the plane, fixes the spin direction according to Eq. (6).

\section{B. Spin precession in a quantum wire due to the spin-orbit interactions}

Let us assume that an electron moves in a onedimensional (1D) ballistic quantum wire along an arbitrary $\theta$ direction and subjected to spin-orbit interactions. Moreover, we neglect the subband hybridization, induced by the spinorbit coupling, assuming that the quantum wire is a truly 1D system because the spin-precession length $\pi / k_{S O}$ is much larger than the wire width. ${ }^{20-22}$ Within our approximation the spin-splitted bands have the orbital parts given by $e^{i k_{ \pm} r}$ ( $r$ is the coordinate along the $\theta$ direction).

In order to calculate the spin-orbit precession along the wire direction, we proceed in the following way (see also van Veehuizen et $a .^{23}$ ). First of all we project an arbitrary input spin state in $r=0$,

$$
|\psi(0)\rangle=\left|\begin{array}{l}
a \\
b
\end{array}\right|
$$

on $\chi_{ \pm}$spinors, obtaining

$$
\left\langle\chi_{+} \mid \psi(0)\right\rangle=a c_{+}+b s_{+} ; \quad\left\langle\chi_{-} \mid \psi(0)\right\rangle=-a s_{+}+b c_{+},
$$

where

$$
c_{+}=\cos \nu_{+} \quad \text { and } \quad s_{+}=\sin \nu_{+} .
$$

Then, after a displacement $L$ along the $\theta$ direction, the electron will be in the state $|\psi(L)\rangle$, given by

$$
|\psi(L)\rangle=e^{i k_{+} L}\left(a c_{+}+b s_{+}\right)\left|\chi_{+}\right\rangle+e^{i k_{-} L}\left(-a s_{+}+b c_{+}\right)\left|\chi_{-}\right\rangle .
$$

It is easy to show that $|\psi(L)\rangle$ can be written in terms of the spin initial state $|\psi(0)\rangle$ :

$$
|\psi(L)\rangle=\left|\begin{array}{cc}
c_{+}^{2} e^{i k_{+} L}+s_{+}^{2} e^{i k_{-} L} & s_{+} c_{+}\left(e^{i k_{+} L}-e^{i k_{-} L}\right) \\
s_{+} c_{+}\left(e^{i k_{+} L}-e^{i k_{-} L}\right) & s_{+}^{2} e^{i k_{+} L}+c_{+}^{2} e^{i k_{-} L}
\end{array}\right| \cdot\left|\begin{array}{c}
a \\
b
\end{array}\right| .
$$

Introducing the spin operator $\mathbf{R}_{S O}$, 


$$
\mathbf{R}_{S O}=\left|\begin{array}{cc}
\cos k_{s o} L-i \cos 2 \nu_{+} \sin k_{s o} L & -i \sin k_{s o} L \sin 2 \nu_{+} \\
-i \sin k_{s o} L \sin 2 \nu_{+} & \cos k_{s o} L+i \cos 2 \nu_{+} \sin k_{s o} L
\end{array}\right|,
$$

Eq. (8) can also be written as

$$
|\psi(L)\rangle=\mathbf{R}_{S O} e^{i \sqrt{\xi^{2}+k_{s o}^{2}}}|\psi(0)\rangle .
$$

In the following, we assume that $\xi^{2} \gg k_{s o}^{2}(\theta)$ because, in the realistic systems, the strength of $\mathrm{SO}, k_{S O} T$, ranges from $0.01 \xi$ to $0.05 \xi$, where $\xi$ is the Fermi wave vector. ${ }^{24}$ Therefore we take the orbital part with $k_{ \pm} \cong \xi \mp k_{s o}$, neglecting terms of the second order in $\xi / k_{s o}$. Then, only the spin operator $\mathbf{R}_{S O}$ depends on the angular position of the wire while the dynamical phase factor becomes equal to $\exp (i \xi L)$. The matrix $\mathbf{R}_{S O}$, actually, describes a geometrical rotation in the $\frac{1}{2}$ spin space around the unitary vector,

$$
\vec{u}=\left(\sin 2 \nu_{+}, 0, \cos 2 \nu_{+}\right)
$$

of the angle $2 k_{s o} L$. In fact, $R_{S O}$ is the representation of the rotation operator, ${ }^{25}$

$$
\mathbf{R}_{S O}=\exp \left(-i k_{s o} L \vec{\sigma} \cdot \vec{u}\right)=\cos k_{s o} L \otimes \mathbf{1}-i \sin k_{s o} L \otimes \vec{\sigma} \cdot \vec{u},
$$

where $\mathbf{1}$ is the unit matrix and $\vec{\sigma}$ is the vector of Pauli matrices.

\section{PERFECT LOCALIZATION DUE TO INTERFERENCE EFFECTS IN LOOPS}

We begin considering the square diamond loop of Fig. 1(b). The dots A and B represent the input and the output leads, respectively. In the following we neglect backscattering effects at the contacts, assuming that the electrons enter A with probability $1 / 2$ in the clockwise path $\mathrm{AB}$ and with
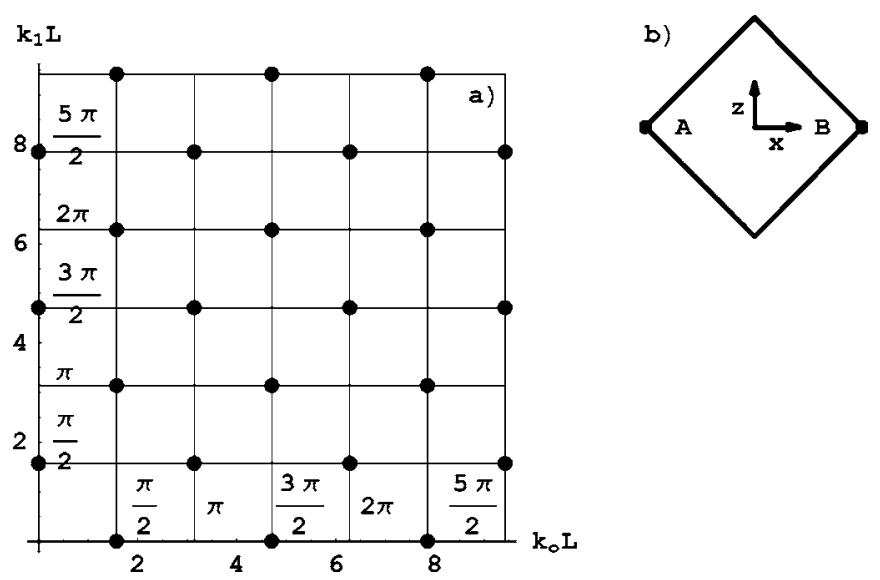

FIG. 1. Perfect localization in the diamond square loop. In (a) there is the contour plot of the transmission as a function of $k_{0} L$ and of the ratio $k_{1} / k_{0}$. (b) shows the zeros of $T$ in the $k_{0} L, k_{1} L$ plane. In (c) is shown the square with the diagonal parallel to the $x$ axis for which the perfect localization occurs. probability $1 / 2$ in the counterclockwise path. The transmission amplitudes matrix $\boldsymbol{\Gamma}$ in $\mathrm{B}$ is

$$
\boldsymbol{\Gamma}=\mathbf{t} e^{i 2 \xi L}
$$

where $t$ is the spin transmission matrix,

$$
\mathbf{t}=\left|\begin{array}{cc}
t_{\uparrow \uparrow} & t_{\uparrow \downarrow} \\
t_{\downarrow \uparrow} & t_{\downarrow \downarrow}
\end{array}\right|
$$

given by the interference between the different spin precessions along the two paths:

$$
\mathbf{t}=\frac{1}{2}\left[\mathbf{R}_{S O}\left(-\frac{\pi}{4}\right) \mathbf{R}_{S O}\left(\frac{\pi}{4}\right)+\mathbf{R}_{S O}\left(\frac{\pi}{4}\right) \mathbf{R}_{S O}\left(-\frac{\pi}{4}\right)\right]
$$

It is simple to show that

$$
t_{\downarrow \downarrow}=t_{\uparrow \uparrow}^{*}=\frac{1}{2}\left(\cos 2 k_{0} L+\cos 2 k_{1} L+i \sqrt{2} \sin 2 k_{0} L\right),
$$

$$
t_{\uparrow \downarrow}=t_{\downarrow \uparrow}=\frac{i}{\sqrt{2}} \sin 2 k_{1} L
$$

Without the Dresselhaus term $\left(k_{1}=0\right)$, the off diagonal elements of the $\mathbf{t}$ matrix vanish and the spin up and spin down states do not interfere. Assuming that the input is an unpolarized statistical mixture,

$$
\rho_{\text {in }}=\frac{1}{2}(|\uparrow\rangle\langle\uparrow|+| \downarrow\rangle\langle\downarrow|)
$$

the output will be described by ${ }^{3}$

$$
\rho_{\text {out }}=\frac{1}{2}\left(T_{\uparrow}|1\rangle\left\langle 1\left|+T_{\downarrow}\right| 2\right\rangle\langle 2|\right),
$$

where $T_{\uparrow}=\left|t_{\uparrow \uparrow}\right|^{2}+\left|t_{\downarrow \uparrow}\right|^{2}$ is the coefficient transmission for an incoming spin up state and $T_{\downarrow}=\left|t_{\uparrow \downarrow}\right|^{2}+\left|t_{\downarrow \downarrow}\right|^{2}$ is that for an incoming spin down state. The spinors in $\rho_{\text {out }}$ are

$$
|1\rangle=\frac{1}{\sqrt{T_{\uparrow}}}\left(\begin{array}{l}
t_{\uparrow \uparrow} \\
t_{\downarrow \uparrow}
\end{array}\right) \quad \text { and } \quad|2\rangle=\frac{1}{\sqrt{T_{\uparrow}}}\left(\begin{array}{l}
t_{\uparrow \downarrow} \\
t_{\downarrow \downarrow}
\end{array}\right)
$$

corresponding to input spin up and down, respectively. Finally the transmission coefficient of the unpolarized electrons is 

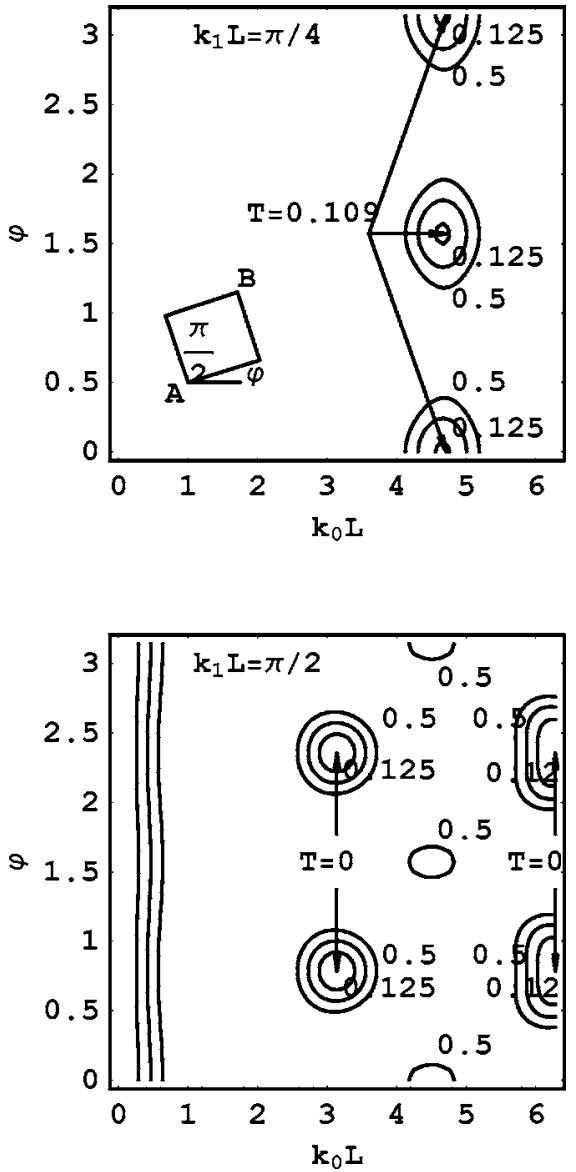

FIG. 2. Contour plots of the transmission coefficient of the rotated square diamond loop as a function of $k_{0} L$ and of the rotation angle $\varphi$ at the two indicated values of $k_{1} L$. The zeros of $T$ appear only for $\varphi=\pi / 4,3 \pi / 4$ for $k_{1} L=\pi / 2$.

$$
\begin{aligned}
T & =\frac{1}{2}\left(T_{\uparrow}+T_{\downarrow}\right)=\frac{1}{2}\left(\left|t_{\uparrow \uparrow}\right|^{2}+\left|t_{\downarrow \downarrow}\right|^{2}+\left|t_{\uparrow \downarrow}\right|^{2}+\left|t_{\downarrow \uparrow}\right|^{2}\right) \\
& =\frac{1}{4}\left(\cos 2 k_{0} L+\cos 2 k_{1} L\right)^{2}+\frac{1}{2}\left(\sin ^{2} 2 k_{0} L+\sin ^{2} 2 k_{1} L\right) .
\end{aligned}
$$

Neglecting the Dresselhaus term $\left(k_{1} L=0\right)$, Eq. (12) provides the known result

$$
T=\cos ^{2} k_{0} L\left(1+\sin ^{2} k_{0} L\right)
$$

that gives perfect localization $(T=0)$ when $k_{0} L=n \pi / 2(n$ $=1,2, \ldots) .{ }^{27}$ When we begin to add gradually the Dresselhaus term, the perfect localization is lost and the zeros of $T$ become transmission minima. Increasing more and more the Dresselhaus SO strength, the perfect localization is recovered when $k_{1} L=\pi / 2$ and a new set of $T=0$ points is obtained corresponding to $k_{0} L=n \pi(n=0,1,2, \ldots)$. As shown in Fig. 1(a), a further increase of $k_{1} L$ generates a regular lattice of $T$ zeros in the $\left(k_{0} L, k_{1} L\right)$ plane, given by

$$
\begin{aligned}
& k_{0} L=n \pi / 2(n=1,2, \ldots), \quad k_{1} L=(m-1) \pi(m=1,2, \ldots) \\
& k_{0} L=(m-1) \pi(m=1,2, \ldots), \quad k_{1} L=n \pi / 2(n=1,2, \ldots) .
\end{aligned}
$$

This result shows that we can get perfect localization in the diamond loop of Fig. 1(b) with both the spin-orbit couplings. On the other hand, we stress that the foregoing result depends strictly on the angular position of the loop with respect to the crystallographic axes of the substrate. Indeed, the geometry studied is somehow special. In order to consider a more general case, we analyze the same square loop rotated by an angle $\varphi$ with respect to the $x$ direction [see the inset of Fig. 2(a)]. The contour plots of $T$ as a function of $\varphi$ and of $k_{0} L$ are given in Fig. 2 for $k_{1} L=\pi / 4$ and $\pi / 2$. For $k_{1} L=\pi / 4$ there is no evidence of $T=0$ points at any $\varphi$. As Fig. 2(a) shows, only transmission minima are present in this case. When $k_{1} L=\pi / 2$ the zeros of $T$ appear at $\varphi=\pi / 4,3 \pi / 4$, which corresponds to align the diagonal of the square loop along the $x$ direction [Fig. 1(b)]. The results confirm that we get perfect localization only for the pair $\left(k_{0} L, k_{1} L\right)$ shown in Fig. 1(a): tilting the square the zeros transform in minima.

In order to make our analysis more complete, we considered also the polygonal loops shown in the insets of Fig. 3: a rhombus and a six sided cell. For the rhombus,
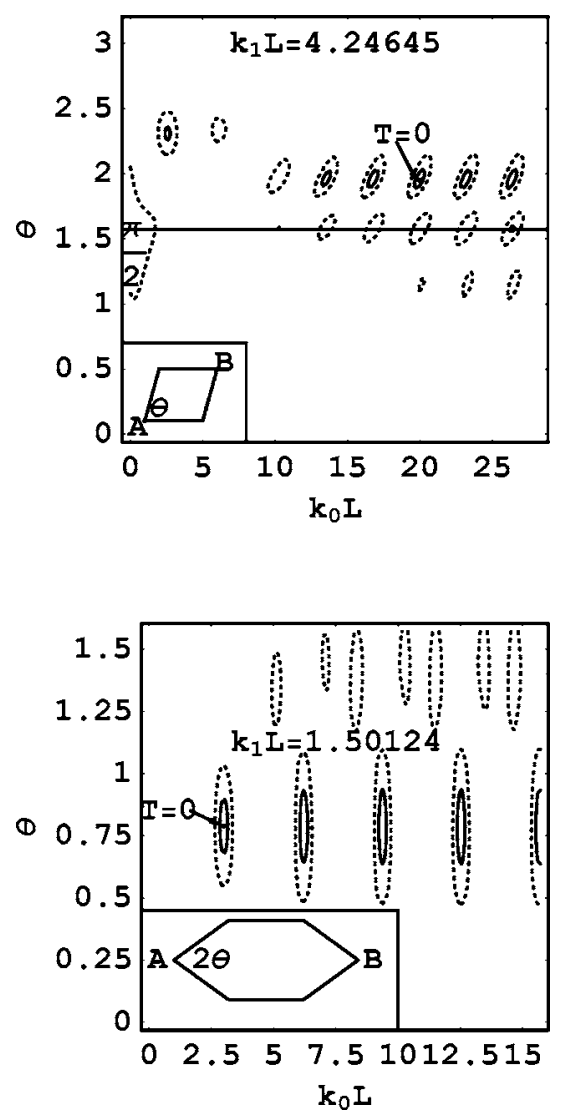

FIG. 3. Contour plots of the transmission coefficient of the rhombus and of the exagonal cell as a function of $k_{0} L$ and of the angular opening $\theta$, at the indicated values of $k_{1} L$ at which an isolated zero of $T$ appear. 
TABLE I. Perfect localization parameters.

\begin{tabular}{ccccccc}
\hline \hline \multicolumn{3}{c}{ Rhombus } & & & \multicolumn{3}{c}{ Exagonal loop } \\
\cline { 1 - 2 } \cline { 5 - 7 }$k_{1} / k_{0}$ & $\theta$ & $k_{0} L$ & & $k_{1} / k_{0}$ & $\theta$ & $k_{0} L$ \\
\hline 0.3126 & 2.0885 & 10.4949 & & 0.4996 & 0.7896 & 3.0048 \\
0.2655 & 2.0313 & 13.6636 & & 0.2500 & 0.7879 & 6.2055 \\
0.2126 & 1.9572 & 19.9739 & & 0.1667 & 0.7867 & 9.3715 \\
0.5015 & 2.2464 & 21.0616 & & 0.2968 & 1.0192 & 10.2090 \\
0.3971 & 2.1721 & 21.8987 & & 0.3749 & 0.8207 & 12.2376 \\
0.2986 & 2.0723 & 22.5772 & & 0.1250 & 0.7862 & 12.5262 \\
0.3620 & 2.1402 & 25.1089 & & & \\
0.2754 & 2.0439 & 25.7455 & & & \\
0.4130 & 2.1853 & 27.5851 & & & \\
\hline \hline
\end{tabular}

$$
\mathbf{t}=\frac{1}{2}\left[\mathbf{R}_{S O}(0) \mathbf{R}_{S O}(\theta)+\mathbf{R}_{S O}(\theta) \mathbf{R}_{S O}(0)\right],
$$

while for the exagonal loop we get

$$
\mathbf{t}=\frac{1}{2}\left[\mathbf{R}_{S O}(\theta) \mathbf{R}_{S O}(0) \mathbf{R}_{S O}(-\theta)+\mathbf{R}_{S O}(-\theta) \mathbf{R}_{S O}(0) \mathbf{R}_{S O} \theta\right] .
$$

From these transmission matrices the transmission coefficient for unpolarized electrons can be obtained, as we have shown in Eq. (12). A careful analysis shows that specific values of $\theta$ exist such that, again, we get the perfect localization $(T=0)$. For such values the vanishing of the transmission appears at some particular pairs of values $\left(k_{1} L, k_{0} L\right)$ that are not connected continuously with the $k_{1}=0$ zeros. In Table I we report the values of $k_{1} / k_{0}, \theta$, and $k_{0} L$ corresponding to a perfect localization $T=0$ for unpolarized electrons. Figure 3 reports contour plots of the transmission as a function of $\theta$ and $k_{0} L$ at the indicated values of $k_{1} L$. The zeros of $T$ appear as particular points at some specific values of the angle $\theta$ and of the spin-orbit strengths. A regular pattern of zeros is a special feature of the square loop configuration of Fig. 1(a) and it is lost for other polygonal loop's shapes.

\section{SO SUPPRESSION OF TRANSMISSION COEFFICIENT MAGNETIC OSCILLATIONS}

In this section we discuss the effect of an external magnetic field $B$ on the transmission properties of a 1D loop under both Rashba and Dresselhaus interactions. We consider, first, a rhombic loop where the injection and the collection nodes coincide with the A node in the inset of Fig. 3(a) (AA configuration). In other words, we are supposing that the collecting point is a splitter in both incoming and outgoing directions, allowing the oscillation of the transmission. This geometry has recently proposed by Koga et al. ${ }^{8}$ to obtain a ballistic spin interferometer. They use the cancellation of the transmission oscillations due to Rashba SO, in the square loop shown in the inset of Fig. 4(a), to achieve an interferometric measure of SO strength $k_{0}$. Since, as we will show in Eq. (15), the transmission coefficient in the presence of a magnetic field can be written in terms of that at a zero magnetic field, we start to discuss the latter case. In the AA configuration the transmission amplitude matrix at a zero magnetic field stems out from the interference between the clockwise $(\mathrm{CW})$ and the counterclockwise $(\mathrm{CCW})$ paths as

$$
\begin{aligned}
\boldsymbol{\Gamma}= & \frac{1}{2}\left[\mathbf{R}_{S O}(x,-\pi, r) \cdot \mathbf{R}_{S O}(x,-\pi+\theta, r)\right. \\
& \times \mathbf{R}_{S O}(x, 0, r) \cdot \mathbf{R}_{S O}(x, \theta, r) \\
& +\mathbf{R}_{S O}(x, \theta-\pi, r) \cdot \mathbf{R}_{S O}(x,-\pi, r) \\
& \left.\times \mathbf{R}_{S O}(x, \theta, r) \cdot \mathbf{R}_{S O}(x, 0, r)\right] e^{i \xi 4 L} \\
= & t_{0}(x, \theta, r) e^{i \xi 4 L} \cdot \mathbf{1}
\end{aligned}
$$

where

$$
\begin{aligned}
\mathbf{R}_{S O}(x, \theta, r)= & \cos x y \otimes \mathbf{1} \\
& -i \sin x y\left(\sin 2 \nu_{+} \otimes \sigma_{x}+\cos 2 \nu_{+} \otimes \sigma_{z}\right)
\end{aligned}
$$

and

$$
x=k_{0} L, \quad y(\theta, r)=\sqrt{1+r^{2}+2 r \sin 2 \theta}=k_{s o}(\theta) / k_{0},
$$

with
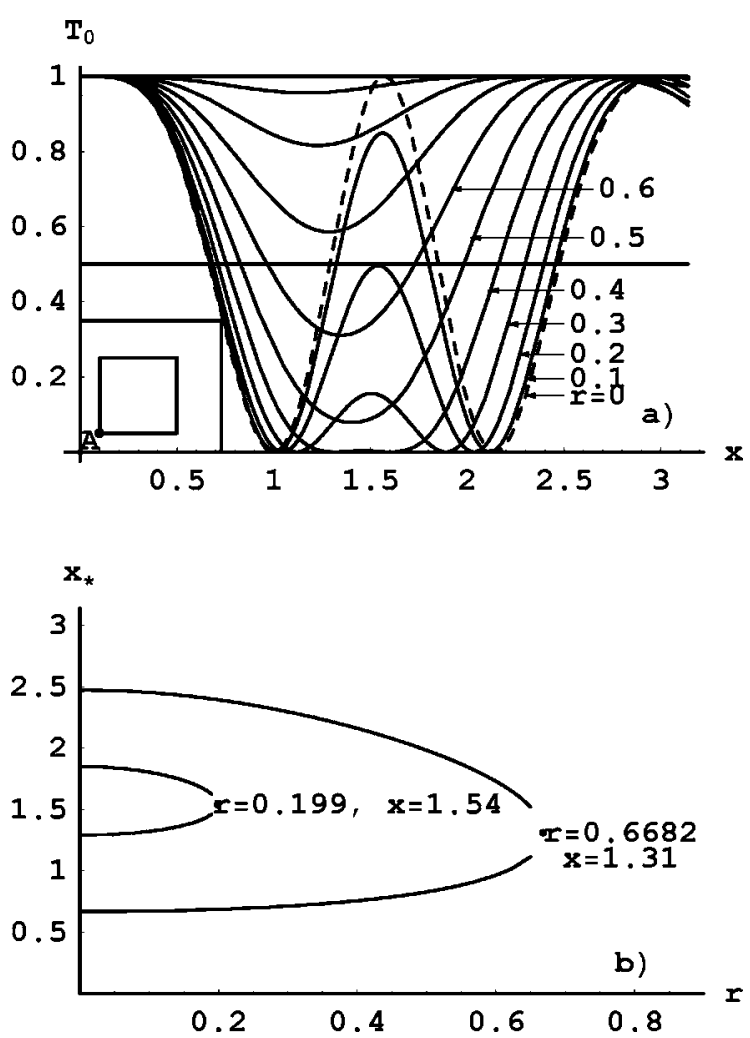

FIG. 4. (a) Transmission coefficient of the square loop $T_{0}$ at a zero magnetic field for the square $(\theta=\pi / 2)$ interferometer (electron enters and exits in A) versus $k_{0} L$ at the indicated values of $r$ $=k_{1} / k_{0}$. (b) A plot of the value $x_{*}$ of $k_{0} L$ as a function of $r$ for which $T_{0}=1 / 2$ on the transmission oscillations are suppressed. 


$$
r=k_{1} / k_{0} \quad \text { and } \quad \nu_{+}=\arctan \frac{\cos \theta+r \sin \theta-y}{\sin \theta+r \cos \theta} .
$$

It is worth noting that the input spin state is conserved and the transmission coefficient,

$$
T_{0}(x, \theta, r)=t_{0}^{2}(x, \theta, r),
$$

is plotted in Fig. 4(a) for $\theta=\pi / 2$ and in Fig. 5(a) for $\theta$ $=\pi / 4$.

In the presence of a magnetic flux, the matrix of the transmitted amplitudes is no longer diagonal and becomes

$$
\begin{aligned}
\boldsymbol{\Gamma}= & \frac{1}{2}\left[\mathbf{R}_{S O}(x,-\pi, r) \cdot \mathbf{R}_{S O}(x,-\pi+\theta, r) \cdot \mathbf{R}_{S O}(x, 0, r) \cdot \mathbf{R}_{S O}(x, \theta, r) e^{i \phi / 2}\right. \\
& \left.+\mathbf{R}_{S O}(x, \theta-\pi, r) \cdot \mathbf{R}_{S O}(x,-\pi, r) \cdot \mathbf{R}_{S O}(x, \theta, r) \cdot \mathbf{R}_{S O}(x, 0, r) e^{-i \phi / 2}\right] e^{i \xi 4 L} \\
= & \left|\begin{array}{ll}
t_{\uparrow \uparrow}(x, \theta, r, \phi) & t_{\uparrow \downarrow}(x, \theta, r, \phi) \\
t_{\downarrow \uparrow}(x, \theta, r, \phi) & t_{\downarrow \downarrow}(x, \theta, r, \phi)
\end{array}\right| e^{i \xi 4 L},
\end{aligned}
$$

with

$$
\begin{gathered}
t_{\uparrow \uparrow} \neq t_{\downarrow \downarrow}, \\
t_{\downarrow \uparrow}=t_{\uparrow \downarrow}^{*} \neq 0 .
\end{gathered}
$$

In Eq. (14) the rhombus (with area $S$ ) is threaded by a magnetic flux $\Phi=B S=\phi \Phi_{0}$, where $\Phi_{0}=h / 2 e$ is the magnetic flux half quanta. The input spin state is no more conserved: the interference between $\mathrm{CW}$ and $\mathrm{CCW}$ paths is able to rotate the spin. The transmission coefficient for unpolarized electrons can be, then, written as

$$
\begin{aligned}
T(x, \theta, r, \phi) & =\frac{1}{2}\left(\left|t_{\uparrow \uparrow}\right|^{2}+\left|t_{\downarrow \uparrow}\right|^{2}+\left|t_{\uparrow \downarrow}\right|^{2}+\left|t_{\downarrow \downarrow}\right|^{2}\right) \\
& =\frac{1}{2}+\left(T_{0}(x, \theta, r)-\frac{1}{2}\right) \cos \phi .
\end{aligned}
$$

As already mentioned, the transmission oscillations are given by the term $\cos \phi$, whose prefactor contains the zero field transmission $T_{0}$, that is all we need to perform the analysis of the magnetic field effects. For a square loop $(\theta=\pi / 2)$ and without the Dresselhaus term $\left(k_{1} L=0\right)$, we recover the known result by Koga et al., ${ }^{8}$

$$
T_{0}\left(x, \frac{\pi}{2}, 0\right)=\left(\cos ^{2} x+\cos 2 x \sin ^{2} x\right)^{2},
$$

which is plotted in Fig. 4(a) (dashed curve). The perfect localization $(T=0)$ is obtained when $x=\pi / 2, \pi$ at $\phi=\pi$. Equation (15) shows that when $T_{0}=1 / 2$, the transmission oscillations are suppressed. On the other hand, the transmission $T$ assumes the same constant value $1 / 2$ when $\phi=\pi / 2,3 \pi / 2$ and, at these magnetic fluxes, the modulation of the transmission due to SO couplings is cancelled. Koga, Sekine, and Nitta $^{9}$ have realized experimentally a Rashba ballistic spin interferometer using a network of square loops. They measured the conductivity $s$ varying the magnetic field and controlling the strength of the Rashba term by means of a gate voltage. Assuming that the conductivity, in the ballistic re- gime, is proportional to the transmission coefficient (15). They searched the values of $x$ for which $s$ becomes independent on the magnetic field $B$ in a range around $B=0$, and from these values they obtained a measure of Rashba SO strength $k_{0}$.

The zero field transmission when also the Dresselhaus term is added (for the square loop) is shown in Fig. 4. Also, in this case the transmission oscillation is suppressed at the $x_{*}(r)$ values for which

$$
T_{0}\left(x_{*}, \theta, r\right)=\frac{1}{2}
$$

Figure 4(b) shows the values of $x_{*}=k_{0} L$ at which the suppression of transmission oscillations is obtained as a function of the ratio between the Dresselhaus and Rashba strength, $r$. Increasing $r$ the period of $T_{0}$ decreases from the value $\pi$ at $r=0$ to lower values. The two zeros of $T$ approach each other and disappear at $x=1.451$ for $r=0.414213$. For $k_{1}=k_{0}$, $T_{0}(x, \pi / 2,1)=1$ and the transmission coefficient becomes independent of the spin-orbit coupling. Figure 4(b) shows that for $r<0.199$ we have four suppression points that become two when $0.199<r<0.668$. The cancellation of transmission oscillations is not possible for greater values of Dresselhaus strength $(r>0.668)$. This analysis shows how relevant the inclusion of the Dresselhaus term is in order to describe in a proper way the transmission oscillations suppression. Furthermore, our study allows an extension of the ballistic spin interferometric technique developed by Koga et al. ${ }^{8}$ that could be used also to measure the ratio between the Rashba and Dresselhaus terms.

To investigate if the transmission oscillations suppression depends on the shape of the interferometer we have taken into account a different rhombus geometry with $\theta=\pi / 4$. Figure 5(a) shows the transmission at the zero field, and Fig. 5(b) shows how the suppression points change with $r$. The cancellation of the oscillation is still present, though the pairs of values at which the suppression occurs $\left(k_{0}, k_{1}\right)$ change, 

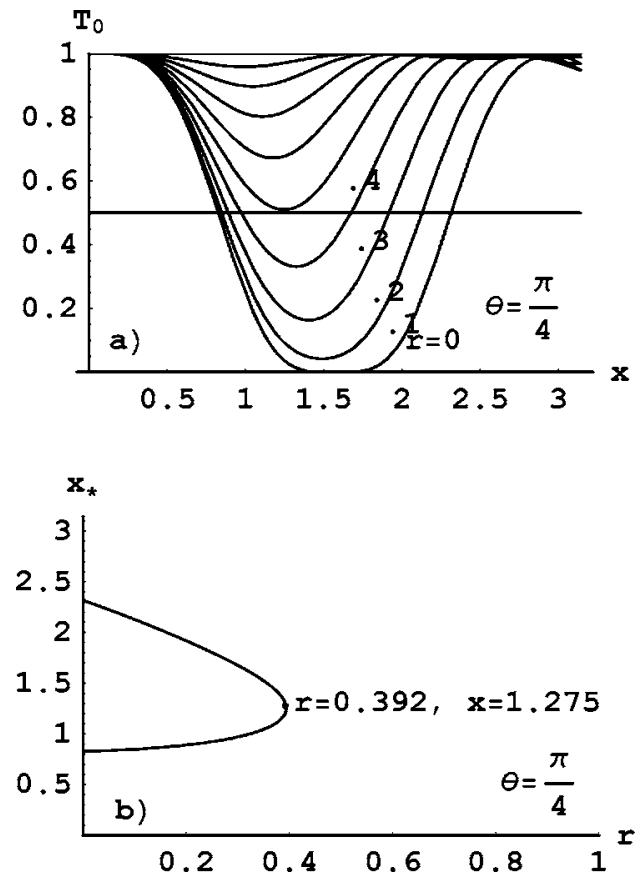

FIG. 5. The same plots of Fig. 4 for a rhombus with an angular opening $\theta$ of $\pi / 4$.

modifying the shape. The suppression remains also when the loop is rotated with respect to the substrate.

To conclude, the analysis of the magnetic field effects let us consider what happens if the electrons are injected in the node $\mathrm{A}$ and collected in the opposite node $\mathrm{B}$, traversing the square loop ( $\mathrm{AB}$ configuration). In this case the transmission amplitudes matrix is given by

$$
\begin{aligned}
\boldsymbol{\Gamma}= & \frac{1}{2}\left[\mathbf{R}_{S O}(x, \pi / 2, r) \cdot \mathbf{R}_{S O}(x, 0, r) e^{i \phi / 4}\right. \\
& \left.+\mathbf{R}_{S O}(x, 0, r) \cdot \mathbf{R}_{S O}(x, \pi / 2, r) e^{-i \phi / 4}\right] e^{i 2 \xi L} \\
= & \left|\begin{array}{lr}
t_{B \uparrow \uparrow} & t_{B \uparrow \downarrow} \\
t_{B \downarrow \uparrow} & t_{B \downarrow \downarrow}
\end{array}\right| e^{i 2 \xi L} .
\end{aligned}
$$

For unpolarized electrons, the transmission coefficient becomes

$$
\begin{aligned}
T_{B}(x, r, \phi) & =\frac{1}{2}\left(\left|t_{B \uparrow \uparrow}\right|^{2}+\left|t_{B \downarrow \downarrow}\right|^{2}+\left|t_{B \uparrow \downarrow}\right|^{2}+\left|t_{B \downarrow \uparrow}\right|^{2}\right) \\
& =\frac{1}{2}+\left(T_{B}(x, r, 0)-\frac{1}{2}\right) \cos \frac{\phi}{2} .
\end{aligned}
$$

The factor $\cos (\phi / 2)$ describes the Aharonov-Bohm oscillations, ${ }^{26}$ which present a double period with respect to the oscillations that we have found in the AA configuration, and, again, his prefactor is fixed by the zero field transmission $T_{B}(x, r, 0)$ that regulates the amplitude of $\mathrm{AB}$ oscillation. This quantity is plotted in Fig. 6(a). As for the foregoing AA configuration, $T_{B}(x, r, 0)=1 / 2$ implies that $T_{B}(x, r, \phi)=1 / 2$ for any $\phi$, and the ratio $r=k_{1} / k_{0}$ can be fixed in such a way that the $\mathrm{AB}$ oscillations are cancelled. Therefore, the suppression takes place at $x$ values satisfying the equation
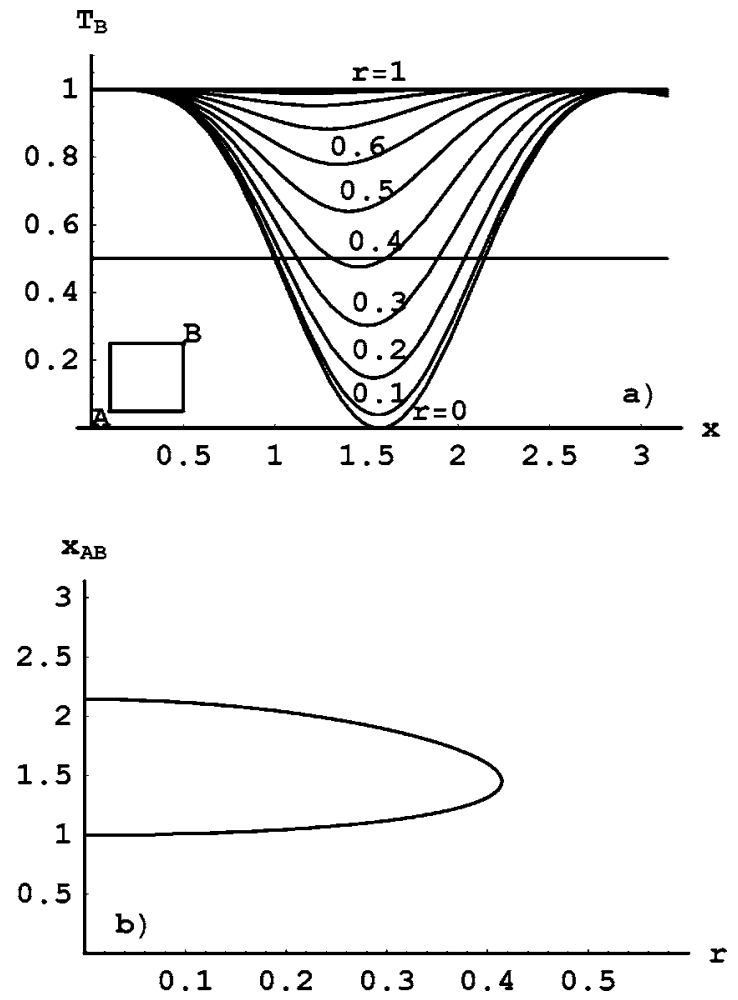

FIG. 6. The suppression of the Aharonov-Bohm oscillations in the square loop (the electrons enter in A and are collected in B). The solutions $x(r)$ of the equation $T_{A B}[x(r), r, 0]=1 / 2$ are shown in (b).

$$
T_{B}\left(x_{A B}(r), r, 0\right)=\frac{1}{2} .
$$

The behavior of the $\mathrm{AB}$ square configuration is shown in Fig. 6.

\section{CONCLUDING REMARKS}

In conclusion, we have studied the interference effects due to the Rashba and the Dresselhaus SO interactions in quantum wires forming polygonal loops. The spin precession along the sides of the loop gives rise to perfect localization (zero transmission coefficient) at particular values of the pair $\left(k_{1} L, k_{0} L\right)$. For the square diamond loop we achieve the perfect localization for pairs $\left(k_{0} L, k_{1} L\right)$ belonging to a square lattice that is symmetrical with respect to the two SO strengths $k_{0}$ and $k_{1}$. The periodic pattern of the transmission zeros $^{27}$ obtained with only the Rashba SO interaction, ${ }^{27}$ is preserved, adding Dresselhaus SO coupling. The configuration with the square diagonal parallel to the $x$ axis (in the [010] crystallographic direction) is a special case and when the square is rotated in the $x-z$ plane the zeros of $T$ transform in minima, and the perfect localization is lost. We have studied other two geometries: a rhombus and an exagonal cell. For both cases pairs $\left(k_{0} L, k_{1} L\right)$ exist that give the perfect localization only for a specific shape (we characterize the shape with an angular opening $\theta$ ). We have found triplets $\left(\theta, k_{0} L, k_{1} L\right)$ that give transmission zeros. This behavior suggests that the perfect localization in a circular loop is not 
easy to predict. In particular, the procedure discussed in Ref. 27 in the case of Rashba coupling, where perfect localization in a circle is obtained as a limit of a succession of regular polygons, cannot be applied in the same way. The perfect localization on a circle with both the SO couplings will be a matter of future research. ${ }^{28}$

When the loop is plunged in an external magnetic field, the transmission coefficient oscillates with the magnetic flux passing through the loop. The amplitude of this oscillation depends on the strengths of the two SO couplings. Injecting and collecting the electrons at the same loop node (the interfering paths are self-intersecting ones), the 1D loop behaves as a ballistic spin interferometer. With this configuration the transmission oscillations appear and, in the presence of Rashba SO, they are suppressed for some particular values of $k_{0} L{ }^{8}$ We have considered an interferometer with the shape of a rhombus with both the SO interactions. The suppression appears at $k_{0} L$ values that depend on the ratio $k_{1} / k_{0}$, so that the interferometric experimental technique of Koga et al. ${ }^{9}$ could be used to measure not only the $k_{0}$ value but also the ratio $k_{1} / k_{0}$. Another kind of magnetic modulation of the transmission coefficient are the $\mathrm{AB}$ oscillations whose period is the double of the foregoing oscillations. They appear when the electrons are injected and collected at opposite nodes of the loop and the interfering paths of equal length surround the loop area. Again the presence of the Dresselhaus coupling can regulate the amplitude of these oscillations.

Our results concern a single loop. When the loops are arranged in a quantum network the transport properties through the system may change as discussed, for the Rashba SO case in Refs. 13 and 14. We also expect that the use of more realistic boundary conditions could be important, for example, a finite coupling with leads can give resonances representing quasibound states within the loop.

To conclude we briefly discuss the consequences of higher order winding contributions and backscattering. The simplest way to deal with this question is to combine the multiple scattering against the injection node and the collector node incoherently. ${ }^{29}$ Then the single scattering event can be characterized with a classical probability. We identify the probability that the electron leaves a node with the transmission coefficients $T$ that we have calculated before, the classical reflection probability being $R=1-T$. The round trips can be arranged into a geometrical series: ${ }^{29}$ the sum of the individual transmission probability gives the composite transmission probability $\mathcal{P}$,

$$
\mathcal{P}=\frac{T^{2}}{1-R^{2}}=\frac{T}{2-T} .
$$

We note that $T=0,1$ implies that also $\mathcal{P}=0,1$. The total transmission probability $\mathcal{P}$ keeps the periodicity in $\phi$, although the dependence on $\phi$ is no more simply $\cos \phi$ or $\cos 2 \phi$ as before. Therefore, this assumption of incoherence predicts that the perfect localization and the suppression of transmission oscillations are not spoiled by incoherent multiple scattering. The transmission probability $\mathcal{P}$ becomes independent on $\phi$ at some particular values $k_{0} L$ in the same way as $T$, with the same dependence on the ratio $r=k_{1} / k_{0}$, but the value of $\mathcal{P}$ at the suppression lowers from $1 / 2$ to $1 / 3$.

\section{ACKNOWLEDGMENTS}

We acknowledge Dario Bercioux for a critical reading of the manuscript. We warmly thank Diego Frustaglia for useful suggestions to clarify some point of the paper.
${ }^{1}$ Semiconductor Spintronics and Quantum Computation, edited by D. D. Awschalom, D. Loss, and N. Samarth (Springer-Verlag, Berlin, 2002).

${ }^{2}$ S. Datta and B. Das, Appl. Phys. Lett. 56, 665 (1990).

${ }^{3}$ V. Marigliano Ramaglia, D. Bercioux, V. Cataudella, G. De Filippis, and C. A. Perroni, J. Phys.: Condens. Matter 16, 9143 (2004)

${ }^{4}$ T. Koga, J. Nitta, H. Takayanagi, and S. Datta, Phys. Rev. Lett. 88, 126601 (2002).

${ }^{5}$ M. G. Pala, M. Governale, J. König, and U. Zülicke, Europhys. Lett. 65, 850 (2004).

${ }^{6}$ M. Governale, F. Taddei, and R. Fazio, Phys. Rev. B 68, 155324 (2003).

${ }^{7}$ E. I. Rashba, Sov. Phys. Solid State 2, 1109 (1960).

${ }^{8}$ T. Koga, J. Nitta, and M. van Veenhuizen, Phys. Rev. B 70, 161302(R) (2004).

${ }^{9}$ T. Koga, Y. Sekine, and J. Nitta, cond-mat/0504743 (unpublished).

${ }^{10}$ J. Nitta and T. Koga, J. Supercond. 16, 689 (2003).

${ }^{11}$ B. Al'tshuler, A. G. Aronov, and B. Spivak, Pis'ma Zh. Eksp. Teor. Fiz. 33, 101 (1981) [JETP Lett. 33, 94 (1981)].

${ }^{12}$ D. Frustaglia and K. Richter, Phys. Rev. B 69, 235310 (2004).

${ }^{13}$ D. Bercioux, M. Governale, V. Cataudella, and V. M. Ramaglia,
Phys. Rev. Lett. 93, 056802 (2004).

${ }^{14}$ D. Bercioux, M. Governale, V. Cataudella, and V. M. Ramaglia, Phys. Rev. B 72, 075305 (2005).

${ }^{15}$ G. Dresselhaus, Phys. Rev. 100, 580 (1955).

${ }^{16}$ S. D. Ganichev, V. V. Belkov, L. E. Golub, E. L. Ivchenko, P. Schneider, S. Giglberger, J. Eroms, J. De Boeck, G. Borghs, W. Wegscheider, D. Weiss, and W. Prettl, Phys. Rev. Lett. 92, 256601 (2004).

${ }^{17}$ S. D. Ganichev, E. L. Ivchenko, V. V. Bel'kov, S. A. Tarasenko, M. Sollinger, D. Weiss, W. Wegscheider, and W. Prettl, Nature (London) 417, 153 (2002).

${ }^{18}$ J. Schliemann, J. C. Egues, and D. Loss, Phys. Rev. Lett. 90, 146801 (2003).

${ }^{19}$ V. Marigliano Ramaglia, D. Bercioux, V. Cataudella, G. De Filippis, C. A. Perroni, and F. Ventriglia, Eur. Phys. J. B 36, 365 (2003).

${ }^{20}$ The limit of this approximation has been studied in Refs. 21 and 22.

${ }^{21}$ F. Mireles and G. Kirczenow, Phys. Rev. B 64, 024426 (2001).

${ }^{22}$ M. Governale and U. Zülicke, Phys. Rev. B 66, 073311 (2002).

${ }^{23}$ M. van Veehuisen, T. Koga, and J. Nitta, cond-mat/0412609 (unpublished).

${ }^{24}$ J. Nitta, T. Akazaki, H. Takayanagi, and T. Enoki, Phys. Rev. 
Lett. 78, 1335 (1997).

${ }^{25}$ Mécanique Quantique, edited by C. Cohen-Tannoudji, B. Diu, and F. Laloë (Hermann, Paris, 1980), Tome II, p. 974.

${ }^{26}$ Y. Aharonov and D. Bohm, Phys. Rev. 115, 485 (1959).

${ }^{27}$ D. Bercioux, D. Frustaglia, and M. Governale, Phys. Rev. B 72,
113310 (2005).

${ }^{28}$ The same result for $T$ is obtained, including only the Dresselhaus term $\left(k_{0}=0\right)$ and replacing in Eq. (13) $k_{0}$ with $k_{1}$.

${ }^{29}$ Electronic Transport in Mesoscopic System, edited by S. Datta (Cambridge University Press, Cambridge, 1995). 